\title{
Visible Light Formation Process
}

\author{
Zhe Yin \\ Department of Mathematics, Yanbian University, China
}

\begin{abstract}
In this paper, we use the gravitational wave theory to elucidate the propagation pattern of light between the electrons of the gravitational waves. The light has the entanglement between the periodic and the optical blocks with the same properties. The degree of entanglement between the light blocks is expressed by relative energy. Each photoperiod contains four sub-process morphologies, each sub-process morphology, forming a separate, electron-group and entangled form of relative energy set.
\end{abstract}

Keywords: light; gravitational wave; wavelength

\section{Introduction}

We proposed in 2010 that substances change to the direction of increase of atomic weight and molecular weight [1]. And we proposed that spiral trajectory of gravitational waves [2] and the variable force field is the condition of energy conversion in February 2016[3,4]. We also proposed that the motion trajectory of the electrons, proposed the law of motion that, spiral trajectory gravitational wave produces entanglement in the process of convergence and divergence, increases the energy, in the trajectory of Mobius', and spiral gravitational wave convergences inwards in March 2016 [3,4,7]. Inward convergence is equivalent that the centripetal force is greater than buoyancy and other external field forces, outward divergence equivalent buoyancy and other external field force is greater than the centripetal force.

The following description of the use of gravitational wave theory, to elucidate the propagation pattern of light between the electrons of the gravitational waves. The light has the entanglement between the periodic and the optical blocks with the same properties. The degree of entanglement between the light blocks is expressed by relative energy. Each photoperiod contains four sub-process morphologies, each sub-process morphology, forming a separate, electron-group and entangled form of relative energy set.

\section{Theoretical preparation and definition preparation[11]}

\subsection{Generation and propagation trajectory theorem of gravitational waves}

Theorem 1: (Gravitational waves existence theory) [3,4,5,6,7]: There are A, B two points. A is the wave source of gravitational field, and $\mathrm{B}$ is a point in the gravitational field. The existence of energy rotational motion (including proton, neutron, atomic nucleus or planet) at $\mathrm{A}$ is the necessary and sufficient condition of the existence of gravitational waves at B. Direction is the bidirection of the path tangent at point B, and the limit of the convergence direction is A point.

Prove : Let say the distance of A and B is $\mathrm{r}$, if A, B are stationary points, it only exists the physical factor of "distance r", do not form elements of waves. Only the rotational movement can generate speed and energy. That is the speed of B point $\mathrm{Vb}$ and the angular velocity of A point $d \theta$ have a functional relationship.

$$
\begin{aligned}
& V b=f(d \theta, r), \text { when time } \Delta t \rightarrow 0, \text { angular velocity } \Delta \theta \rightarrow 0, \text { and } \\
& V b=\frac{d r}{d t}=\lim _{\Delta \theta \rightarrow 0} \frac{[f(\theta+\Delta \theta), r]-[f(\theta), r]}{\Delta \theta},
\end{aligned}
$$

We have a conclusion that $\mathrm{A}$ is the wave source of gravitation, and point $\mathrm{B}$ exists the gravitational wave emitted from wave source A.

2.2 Theorem 2(Gravitational wave stability theory) $[3,4,5,6,7]$ : A is a gravitational wave source, B is a point in gravitational field of A. The necessary and sufficient conditions of stable gravitational wave at B point is :

$$
\frac{d r}{d \theta}=b \quad, \quad(\mathrm{~b} \text { is a constant) } .
$$

Prove : According to Theory 1 ,

$$
V b=\frac{d r}{d t}=\lim _{\Delta \theta \rightarrow 0} \frac{[f(\theta+\Delta \theta), r]-[f(\theta), r]}{\Delta \theta}
$$


The necessary and sufficient conditions of stable gravitational wave is that the speed of B point is stable ;

The necessary and sufficient conditions of stable speed at B point are that the angular velocity of A point is stable; The necessary and sufficient conditions of stable angular velocity of A point is that the angular velocity of A point and speed of B point is proportional.

$$
\begin{aligned}
& \text { That is, } \\
& \frac{V b}{d \theta}=\frac{d r}{d \theta}=b
\end{aligned}
$$

and,

$$
r=a+b \theta \text {, }
$$

formula (1) is the equations of gravitational waves, that is the track of gravitational waves.

Inference of theorem 2 : If a gravitational wave track meet Archimedean spiral, this must be stable gravitational waves.

There is, $r=a+b \theta$

$\mathrm{r}$ is the distance of $\mathrm{A}$ to $\mathrm{B}$; $\mathrm{a}$ is the spiral length of $\mathrm{A}$ to $\mathrm{B}$; $\mathrm{b}$ is the distance between the spirals.

\section{The Propagation Theorem of light Periodicity and light Entanglement of Zhe Yin 's}

According to the gravitational wave equation, the periodic law of light is obtained. Gravitational waves are the propagation of light. Light is the same form of electronic, entangled particles in the form of transmission. Photons have relative energy and quality. Through the electronic entanglement, supplement and increase the energy, gradually increase the wavelength.

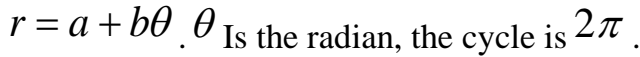

Theorem 1: The wave of light is periodic and its period is $2 \pi$.

According to the independence, symmetry, monotonic principle, light has only four forms. (Four sub-process morphologies of the photoperiod).

Theorem 2: Four independent morphological equations of light:

The first sub-process morph equation: $r=a+b(2 k \pi+0), k=0,1,2,3, \ldots$;

The second sub-process morph equation: $r=a+b\left(2 k \pi+\frac{\pi}{2}\right), k=0,1,2,3, \ldots$;

The third sub-process morph equation: $r=a+b(2 k \pi+\pi), k=0,1,2,3, \ldots$;

The fourth sub-process morph equation:

$$
r=a+b\left(2 k \pi+\frac{3 \pi}{2}\right), k=0,1,2,3, \ldots
$$

As in different environments, hydrogen atoms can be positively charged or negatively charged, the electron entangled state (photon) can be positively charged or negatively charged. Each sub-process of electrons forms a separate set of photonics. Form and share relative energy.

Anywhere on earth, four kinds of photon clusters coexist. The relative energy of the photon is proportional to the wavelength.

Theorem 3: a point on the earth, with a certain relative energy, four kinds of photon collection, the wavelength range is:

The wavelength range of the first sub-process form: [380,495], unit $\mathbf{n m}$;

The Wavelength range of the second sub-process form: [476,620], unit nm;

The wavelength range of the third sub-process form: [570,750], unit nm;

The wavelength range of the fourth sub-process form: $[590,790]$ unit $\mathbf{n m}$.

Visible light in the wavelength range $[380,450]$ shows purple, because the double entanglement is exactly equal to the red light wavelength. (Other does not exist).

The relative energy represents the degree of entanglement and the total amount of photons, and the color represents the density of the photon. Density determines the color of any substance. The density is proportional to the reflectivity of light.

Theorem 4: The size of the entanglement of each sub-process to meet the normal distribution, the closer the wavelength, the better the entanglement, the greater the relative energy.

DOI: $10.9790 / 4861-0902013739 \quad$ www.iosrjournals.org $38 \mid$ Page


Lyman series, Balmer series, Paschen series are different sub-process description of the form.

\section{Conclusion}

In this paper, the use of gravitational wave properties, clarify the light entanglement and relative energy formation process. Clarify the principle of the four forms of light. The cyclical principle of light not only the general light source of the sun, the study of high energy light source, will play its due role.

\section{References}

[1]. Zhe Yin,(2010).The Co-Planarity and Symmetry Principle of Earthquake occurrence, International Journal of Geosciences,pp:38-43.

[2]. j. Weber, $(1959,2016)$, Gravitational Wave, Laser interferometer gravitational wave observatory, 2016.2.11.23:30.

[3]. Zhe Yin,dongxu liu.(2016). Electron trajectory, International Journal of Applied Science and Mathematics,3( 2),56-57.

[4]. Zhe Yin, Dongxu L (2016). Causes of sound wave on the moon. Int. J. Eng. Appl. Sci. 3(2):56-58.

[5]. Zhe Yin (Yin Z), Zhang W, Bie H (2016). Zhe yin's Theorem of Molecular Biology. Acad. J. Sci. Res. 4(4): 099-102.

[6]. Zhe Yin,(2016). Zhe yin's energy and Wave theorem, International Journal of Engineering and Advanced Research Technology (IJEART)ISSN: 2454-9290, 2(3):44-45.

[7]. Zhe Yin.2017, The Function And Relation of Proton And Neutron. Int J Recent Sci Res. 8(1), pp. 15321-15324 